\title{
Effective Oxidation-Responsive Polyester Nanocarriers for Anti-Inflammatory Drug Delivery
}

\author{
Pan $\mathrm{He}$ (D) $^{\prime}$ \\ Bingtong Tang' \\ Yusheng $\mathrm{Li}^{\prime}$ \\ Yu Zhang ${ }^{2}$ \\ Xinming Liu $^{2}$ \\ Xin Guo' \\ Dong Wang ${ }^{3}$ \\ Peng She $\mathrm{S}^{3,4}$ \\ Chunsheng Xiao (iD ${ }^{2}$
}

'School of Materials Science and Engineering, Changchun University of Science and Technology, Changchun, 130022, People's Republic of China; ${ }^{2}$ Key Laboratory of Polymer Ecomaterials, Changchun Institute of Applied

Chemistry, Chinese Academy of Sciences, Changchun, 130022, People's Republic of China; ${ }^{3}$ Department of Orthopaedics, The Seventh Affiliated Hospital of Sun Yat-sen University, Shenzhen, 518107, People's Republic of China; ${ }^{4}$ Joint Surgery Department, The First Hospital, Jilin University, Changchun, I3002I, People's Republic of China
Correspondence: Pan He; Peng She School of Materials Science and Engineering, Changchun University of Science and Technology, Changchun, 130022, People's Republic of China Tel +86-43I-85583। 88

Fax +86-43I-85583I88

Email hepan@cust.edu.cn; shep@mail. sysu.edu.cn
Background: High levels of oxidants, such as reactive oxygen species (ROS) and reactive nitrogen species (RNS), are typical characteristics of an inflammatory microenvironment and are closely associated with a various inflammatory pathologies, eg, cancer, diabetes, atherosclerosis, and neurodegenerative diseases. Therefore, the delivery of anti-inflammatory drugs by oxidation-responsive smart systems would be an efficient anti-inflammatory strategy that benefits from the selective drug release in an inflammatory site, a lower treatment dose, and minimizes side effects.

Purpose: In this study, we present the feasibility of an oxidation-sensitive PEGylated alternating polyester, methoxyl poly(ethylene glycol)-block-poly(phthalic anhydride-alterglycidyl propargyl ether) (mPEG- $b$-P(PA-alt-GPBAe)), as novel nanocarrier for curcumin (CUR), and explore the application in anti-inflammatory therapy.

Methods: The copolymers used were obtained by combining a click reaction and a ringopening-polymerization method. CUR was loaded by self-assembly. The in vitro drug release, cytotoxicity toward RAW 264.7 cells and cellular uptake were investigated. Furthermore, the anti-inflammatory effects of CUR-loaded polymeric nanoparticles (NPsCUR) were investigated in lipopolysaccharide (LPS)-stimulated RAW 264.7 macrophages and tested in a murine model of ankle inflammation.

Results: Fast drug release from NPs-CUR was observed in trigger of $1 \mathrm{mM} \mathrm{H}_{2} \mathrm{O}_{2}$ in PBS. Compared with NPs and free drugs, the significant anti-inflammatory potential of NPs-CUR was proven in activated RAW 264.7 cells by inhibiting the production of TNF- $\alpha$, IL-1 $\beta$, and IL-6 and increasing the level of an anti-inflammatory cytokine IL-10. Finally, a local injection of NPs-CUR at a dose of $0.25 \mathrm{mg} / \mathrm{kg}$ suppressed the acute ankle inflammatory response in mice by histological observation and further reduced the expression of proinflammatory cytokines in the affected ankle joints compared to that of free CUR.

Conclusion: Both the significant in vitro and in vivo anti-inflammatory results indicated that our oxidation responsive polymeric nanoparticles are promising drug delivery systems for anti-inflammatory therapy.

Keywords: oxidation responsiveness, PEGylated polyesters, nanosized micelles, antiinflammatory therapy, curcumin delivery

\section{Introduction}

Uncontrolled inflammation is closely associated with various pathologies, such as atherosclerosis, asthma, neurodegenerative diseases, and cancer. ${ }^{1,2}$ Recently, in several studies, it was suggested that the major cause of disease severity and death in coronavirus disease 2019 (COVID-19) infected patients was virally 
induced-hyperinflammation. ${ }^{3,4}$ One striking feature of inflammation is the oxidative stress caused by overproduction of reactive oxygen species (ROS). Moreover, ROSderived oxidants, including hydrogen peroxide $\left(\mathrm{H}_{2} \mathrm{O}_{2}\right)$ at the inflammatory site is highly reactive to proteins and DNA, and causes necrosis or apoptosis. ${ }^{5}$ Therefore, it is of significance to scavenge the over-generated oxidants and to target the delivery of anti-inflammatory agents for anti-inflammatory therapy. ${ }^{6}$

Oxidation-responsive polymeric vesicles have been proven to be efficient and sufficient drug delivery systems (DDS) for anti-inflammatory agents, because the polymer carrier reacted with oxidants, down-regulated ROS level and released the incorporated drug in the high oxidizing environment at the inflammatory site. ${ }^{7,8}$ Lee et al prepared $\mathrm{H}_{2} \mathrm{O}_{2}$-responsive polymeric prodrug nanoparticles (NPs) of vanillin with peroxalate ester bonds and acid-labile acetal linkages, which degraded in cells by acid and oxidative stress, released antioxidant and anti-inflammatory drug vanillin simultaneously, and thus inhibited the expression of TNF- $\alpha$ and iNOS in lipopolysaccharide (LPS)-stimulated RAW 264.7 cells. ${ }^{9}$ The authors also developed vanillyl alcohol-based anti-oxidant polymer nanoparticles to treat doxorubicin-induced cardiomyopathy, and macroparticles loaded with Manganese porphyrin for the treatment of acute liver failure in mice. ${ }^{10,11}$ Tirelli's group pioneered in the development of hydrophobic poly (propylene sulphide) (PPS)-based polymeric nanoparticles, which were conversed to hydrophilic polysulphoxide and ultimately polysulphones by $\mathrm{H}_{2} \mathrm{O}_{2}$, leading to oxidationtriggered particle destabilization. ${ }^{12}$ Most interestingly, PPS-based nanoparticles were found to be pharmacologically active materials, which showed ROS-scavenging activity and reduced the production of cytokines by LPSactivated macrophages. ${ }^{13}$ In their recent work, PEGylated PPS nanoparticles were applied for ischemic stroke therapy and showed reduced post-stroke inflammation and damage through scavenging ROS in a mouse model of stroke. ${ }^{14}$ Besides sulfide, polymers containing thioether, thioketal, boronic acid groups and polystannanes exhibited oxidative responses, whereas their biocompatibility and biodegradation properties needed improving as DDS. ${ }^{15-17}$

Curcumin (CUR), 1,7-bis-(4-hydroxy-3-methoxyphenyl)-hepta-1,6-diene-3,5-dione, has received significant attention as a natural phenolic antioxidant and an anticancer drug. Mounting evidence has proven the anti-inflammatory role of CUR and its antiproliferative effects on tumor cells. ${ }^{18}$ In fact, the antitumor properties of CUR are ascribed to its antioxidant and free-radical scavenging capabilities. ${ }^{19}$ However, the extreme hydrophobicity and poor hydrolytic stability of CUR have limited its clinical applications. To this end, various drug delivery platforms have been developed for the incorporation of CUR to improve its water solubility and therapeutic activity. ${ }^{20}$ Sung and colleagues developed dual responsive nanoparticles as CUR carriers that are composed of poly-(1,4-phenyleneactone dimethylene thioketal) and chitosan-derivatives, which disintegrated under oxidative stress and reduced $\mathrm{pH}$ in inflamed conditions. The released CUR reduced the level of excess oxidants in LPS-stimulated macrophages, and exhibited an enhanced anti-inflammatory activity in a mouse model of ankle inflammation. ${ }^{6}$ CURencapsulated yeast $\beta$-glucan particles (GPs) showed enhanced anti-inflammatory potential in vitro, and have been proven to reduce GPs-mediated IL- $1 \beta$ and TNF- $\alpha$ cytokine production in isolated porcine peripheral blood mononuclear cells. ${ }^{21}$ In addition, PEGylated CUR prodrug micelles and cyclodextrin have been used as CUR carriers to improve CUR's solubility, stability, and bioavailability. ${ }^{22}$

Recently, we synthesized a class of PEGylated alternating polyesters by ring-opening alternating copolymerization (ROAP). ${ }^{23-25}$ The hydrophobic thioether pendant groups of one copolymer were selectively oxidized to hydrophilic sulfoxide by $50 \mathrm{mmol}$ of $\mathrm{H}_{2} \mathrm{O}_{2}$, leading to dissociation of the self-assembled micelles and release of the payload. ${ }^{24} \mathrm{To}$ improve the oxidative sensitivity of polymer, phenylboronic acid pinacol ester was conjugated to another PEGylated polyester by combining ROAP using the "click" chemistry reaction. The obtained copolymer, methoxyl poly(ethylene glycol)-block-poly(phthalic anhydride-alter-glycidyl propargyl ether) (mPEG- $b$-P(PA-alt-GPBAe)), could assemble into nanoparticles and encapsulate Doxorubicin with a high loading content. Accelerated drug release was observed in the trigger of $1 \mathrm{mmol}$ of $\mathrm{H}_{2} \mathrm{O}_{2}$ in acidic PBS and an enhanced anticancer effect was achieved both in vitro and in $4 \mathrm{~T} 1$ tumor-bearing mice. ${ }^{25}$

In the present work, the potential of mPEG- $b$-P(PA-altGPBAe) copolymers as oxidation responsive carriers of CUR and their anti-inflammatory effects were evaluated both in vitro and in vivo (Scheme 1). Firstly, the $\mathrm{H}_{2} \mathrm{O}_{2}$ triggered CUR release in PBS, the cytotoxicity, and the cellular uptake were investigated. Subsequently, the inhibition of proinflammatory cytokine production by NPs, free CUR, and CUR-loaded NPs was evaluated in LPSstimulated RAW 264.7 cells by enzyme linked immunosorbent assay (ELISA). Finally, the anti-inflammatory effects of different CUR formulations were tested in 


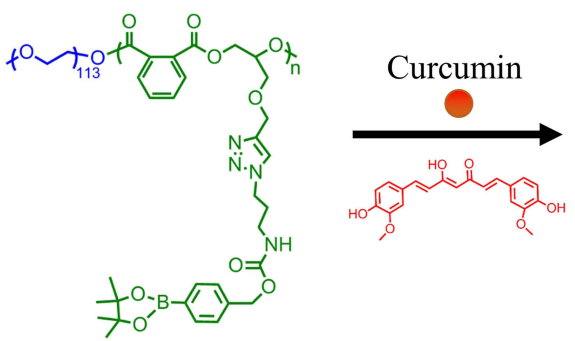

mPEG- $b$-P(PA-alt-GBPAe)

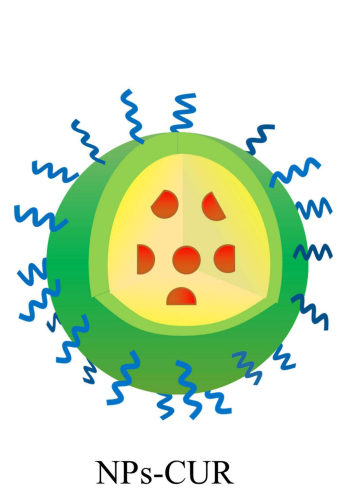

NPs-CUR

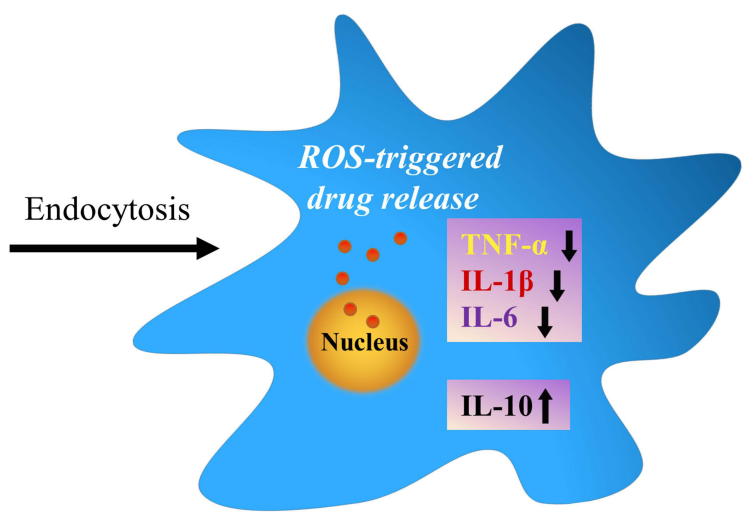

Activated Macrophage

Scheme I Chemical structures of curcumin and its polymeric carrier mPEG-b-P(PA-alt-GPBAe), and a schematic illustration of the anti-inflammatory mechanism of NPsCUR in an activated macrophage model.

a mouse model of ankle inflammation. Due to the $\mathrm{H}_{2} \mathrm{O}_{2-}$ scavenging effects and responsive ability of the PEGylated copolymers, significant anti-inflammatory properties of the CUR-loaded nanoparticles are expected.

\section{Materials and Methods Preparation of Blank Polymeric Nanoparticles (NPs)}

The target oxidation sensitive copolymer mPEG- $b$-P(PAalt-GPBAe) was obtained as described in our previous study. $^{25}$ For the preparation of blank NPs, $25 \mathrm{mg}$ of mPEG- $b$-P(PA-alt-GPBAe) were dissolved in $1.5 \mathrm{~mL}$ of dimethyl formamide (DMF), and then added dropwise into $10 \mathrm{~mL}$ of water. The mixture was stirred for $2 \mathrm{~h}$ at room temperature, and then dialyzed against deionized (DI) water using a dialysis membrane (MWCO $3500 \mathrm{Da}$ ) for 24 h. After DMF was removed, part of the obtained polymeric nanoparticles were diluted and characterized by dynamic light scattering (DLS) on a Zetasizer (Nano-ZS, Malvern Instruments, UK) and by transmission electron microscopy (TEM) on a JEM-1011 transmission electron microscope (JEOL Inc., Tokyo, Japan) with an accelerating voltage of $100 \mathrm{kV}$. The other part of NPs was freezedried and stored.

The oxidation-sensitive behavior of mPEG- $b$-P(PA-altGPBAe) NPs was evaluated by monitoring the size changes by DLS. In brief, NPs in $0.1 \mathrm{mg} / \mathrm{mL}$ were treated with $0.1 \mathrm{~mol} \mathrm{H}_{2} \mathrm{O}_{2}$ and incubated at room temperature for $24 \mathrm{~h}$, subsequently, DLS was performed at 2, 4, 8, 12, and $24 \mathrm{~h}$, respectively. Finally, the morphology of the NPs was analyzed by TEM.

\section{Preparation of CUR-Loaded Nanoparticles and in vitro Drug Release} CUR was purchased from Heowns Biochemical Technology Co., Ltd (Tianjin, China), and was used as received. In brief, $5 \mathrm{mg}$ of CUR and $20 \mathrm{mg}$ of polymer were mixed in $1.5 \mathrm{~mL}$ of DMF under vigorous stirring. Then, CUR-loaded nanoparticles (NPs-CUR) were prepared by the aforementioned dialysis method. Dialysis was conducted in the dark to protect CUR from degradation. NPs-CUR were obtained after lyophilization. The encapsulated CUR content was determined by a UV-vis spectrometer at $\lambda=426 \mathrm{~nm}$ (Lambda 365, PerkinElmer, USA). The drug loading content (DLC) and drug loading efficiency (DLE) were calculated according to the following formulation:

DLC $(\%)=$ (weight of loaded CUR in NPs-CUR /weight of NPs-CUR) $\times 100 \%$

DLE $(\%)=($ weight of loaded CUR/weight of feeding CUR) $\times 100 \%$

The release of oxidation-triggered CUR was performed by dialysis of $5.0 \mathrm{mg}$ of NPs-CUR against $50 \mathrm{~mL}$ of PBS with $1 \mathrm{mmol}$ of $\mathrm{H}_{2} \mathrm{O}_{2}$. At predetermined times, $1 \mathrm{~mL}$ of the released solution was collected and replaced by $1 \mathrm{~mL}$ of fresh PBS containing $\mathrm{H}_{2} \mathrm{O}_{2}$. The concentration of released CUR was measured by a UV-vis spectrometer (UV-1800, Shimadzu, Kyoto, Japan) using a standard curve.

\section{Cell Culture and Cell Viability Studies}

RAW 264.7 cells were purchased from BeNa Culture Collection Company (Beijing, China), and cultured in complete Dulbecco's modified Eagle's medium (DMEM, GIBCO) medium containing $10 \%(\mathrm{~V} / \mathrm{V})$ inactivated fetal 
bovine serum (FBS), streptomycin $\left(50 \mathrm{U} \mathrm{mL}^{-1}\right)$ and penicillin $\left(50 \mathrm{U} \mathrm{mL}^{-1}\right)$ at $5 \% \mathrm{CO}_{2}$ and at $37{ }^{\circ} \mathrm{C}$. MTT, 3-(4,5-Dimethyl-thiazol-2-yl)-2,5-diphenyl tetrazolium bromide, was purchased from Sigma-Aldrich (Shanghai, China). In brief, RAW 264.7 cells in $180.0 \mu \mathrm{L}$ DMEM were seeded into wells of a 96-well-plate at a density of $1.0 \times 10^{4} /$ well and incubated overnight. Then, the supernatant was replaced with DMEM containing NPs-CUR or free CUR at a CUR dose of $1.25 \mu \mathrm{M}$ to $40 \mu \mathrm{M}$, or the equivalent amount of unloaded NPs. After $24 \mathrm{~h}, 20.0 \mu \mathrm{L}$ MTT stock solution ( $5 \mathrm{mg} \mathrm{mL}^{-1}$ ) was added to each well and cells were cultured for another $4 \mathrm{~h}$.

The supernatant of each well was discarded and replaced with $150 \mu \mathrm{L}$ DMSO. After shaking for $10 \mathrm{~min}$ utes, the absorbance of the above-mentioned solution was measured at $490 \mathrm{~nm}\left(\mathrm{~A}_{490}\right)$ on a Bio-Rad 680 microplate reader $(\mathrm{Bio}-\mathrm{Rad}$, Hercules, $\mathrm{CA}$, USA). Cell viability $(\%)=$ $\left(\mathrm{A}_{\text {sample }} / \mathrm{A}_{\text {control }}\right) \times 100 \%$, where $\mathrm{A}_{\text {sample }}$ and $\mathrm{A}_{\text {control }}$ represent the $\mathrm{A}_{490}$ of the sample and control (PBS) wells, respectively.

To evaluate the cytotoxicity toward LPS-activated RAW cells, macrophages were stimulated using an LPS dose of $1.0 \mu \mathrm{g} / \mathrm{mL}$ for $24 \mathrm{~h}$, and were then seeded in 96well-plates for $12 \mathrm{~h}$. Next, DMEM containing three different formulations with a CUR dose of $1.25 \mu \mathrm{M}$ to $40 \mu \mathrm{M}$ were added and cells were incubated for another 24 h. Subsequently, cells were submitted for MTT assay evaluation.

\section{Cellular Uptake/Internalization}

LPS-stimulated RAW 264.7 cells were seeded on a coverslip in 6-well plates at a density of $4 \times 10^{4}$ cells per well, and incubated for $24 \mathrm{~h}$. Subsequently, the supernatant was discarded and replaced with DMEM containing NPs-CUR. After incubation for another $2 \mathrm{~h}$ to $8 \mathrm{~h}$, the cells were washed with PBS and fixed with $4 \%$ formaldehyde for $30 \mathrm{~min}$. Finally, the green fluorescence of CUR $\left(\lambda_{\mathrm{ex}}=\right.$ $456 \mathrm{~nm}$ ) was monitored by confocal laser scanning microscopy. (CLSM, LSM 780; Carl Zeiss, Germany)

\section{In vitro Anti-Inflammatory Effects of NPs-CUR}

RAW 264.7 cells were seeded in 6 -well plates $\left(4 \times 10^{4}\right.$ cells per well), left to adhere overnight, and were incubated for $2 \mathrm{~h}$ with NPs-CUR or free CUR at a CUR dose of $10 \mu \mathrm{M}$, or the equivalent amount of unloaded NPs. Subsequently, macrophages were stimulated with
$1.0 \mu \mathrm{g} / \mathrm{mL}$ of LPS for $24 \mathrm{~h}$. Cells treated with PBS and without LPS treatment served as controls. Supernatant was collected by centrifugation at $3000 \mathrm{rpm}$ for $20 \mathrm{~min}$ at room temperature. Levels of TNF- $\alpha$, IL-1 $\beta$, IL-6, and IL-10 were quantified using an ELISA Kit (Dayou ${ }^{\circledR}$, Dakewe Biotech Co., Ltd.; Shenzhen, China), following the manufacturer's instructions.

\section{In vivo Anti-Inflammatory Effects of NPs-CUR}

Adult female C57BL/6 mice (8 weeks old, 20-25 g) were purchased from the Beijing Vital River Laboratory Animal Technology Co., Ltd (Beijing, China), and were housed in controlled temperature rooms $\left(23 \pm 2{ }^{\circ} \mathrm{C}\right)$, under a $12 / 12 \mathrm{~h}$ light-dark cycle, with access to water and food ad libitum. All animal procedures were performed in accordance with the Guidelines for Care and Use of Laboratory Animals of Jilin University, and approved by the Animal Ethics Committee of Jilin University. Mice were randomly separated into 4 groups (3-5 mice per group). Healthy mice that did not receive any treatment were used as the control group (Control). LPS (2 $\mathrm{mg} / \mathrm{kg}$ ) was injected into the ankles of mice in other 3 groups. Four h later, NPs, free CUR or NPs containing CUR at a dose of $0.25 \mathrm{mg} / \mathrm{kg}$ were injected into one of the inflamed ankles (NPs, CUR and NP-CUR group, respectively); the other inflamed ankle received PBS and represented the PBS group. Six h later, mice were sacrificed. The ankle joints were excised, cleaned with PBS, immersed in $4 \%$ buffered paraformaldehyde overnight, and decalcified using a $10 \%(\mathrm{w} / \mathrm{v})$ EDTA for 20 days. After embedding in paraffin, ankle joint tissue was cut into $5 \mathrm{~mm}$ sections and stained with hematoxylin-eosin (H\&E). Sections were also used for immunofluorescence analysis using antibodies directed against CD86, CD206, iNOS, TNF- $\alpha$, IL-1 $\beta$ and IL-6. H\&E-stained sections were imaged by a Nikon TE2000U microscope for detailed evaluation of inflammation. The levels of cell surface markers, iNOS, and inflammatory cytokines in the tissue were determined by CLSM using a LSM 780 (Carl Zeiss, Jena, Germany). In addition, the relative positive areas of proinflammatory cytokines were measured using ImageJ software (National Institutes of Health, Bethesda, Maryland) for semiquantitative analyses. 


\section{Statistical Analysis}

All experiments were conducted at least in triplicate, and the results are shown as the mean value \pm standard deviation (SD). ${ }^{*} \mathrm{P}<0.05$ was considered statistically significant. $* * \mathrm{P}<0.01$ and $* * * \mathrm{P}<0.001$ were considered highly significant.

\section{Results and Discussion \\ NPs Formation and the Oxidation-Sensitive Behavior}

The detailed synthesis route and the ${ }^{1} \mathrm{H}-\mathrm{NMR}$ spectrum of the target oxidation sensitive polymer used in this study are shown in the supporting information. As illustrated in Figure S1, mPEG- $b$-P(PA-alt-GPBAe) was synthesized by combining the click reaction and a ROAP method using
mPEG-OH as the macroinitiator and a phosphazene base as the catalyst, which was similar to what was used in our previously reported study. ${ }^{24,25}$ All the proton or carbon resonance peaks were assigned in accordance with the proposed structure of mPEG- $b$-P(PA-alt-GPBAe) in the ${ }^{1} \mathrm{H}-\mathrm{NMR}$ and ${ }^{13} \mathrm{C}-\mathrm{NMR}$ spectra (Figure S2 and $\underline{\mathrm{S} 3}$ ). The average molecular weight was determined to be $13.9 \mathrm{kDa}$ with a narrow polydispersity value of 1.06 as determined by gel permeation chromatography (Figure S4). In this study, the obtained amphiphilic copolymer, mPEG- $b$-P (PA-alt-GPBAe), could self-assemble in water into nanosized micelles with a PEG shell and a polyester core, called NP). CUR was encapsulated into NPs with a DLC of $13.2 \%$ and a DLE of $60.7 \%$, respectively. The CURloaded NPs obtained were abbreviated as NPs-CUR. The interaction between the drug and phenyl-containing

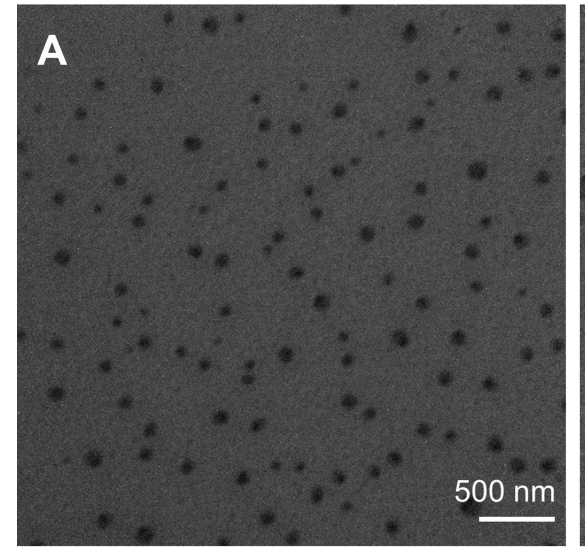

C

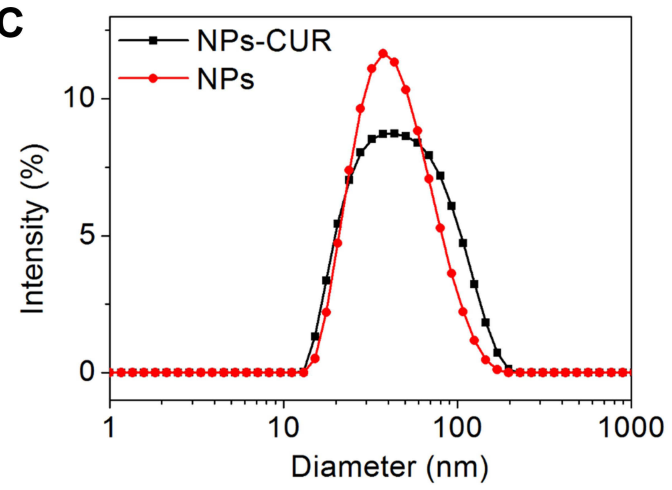

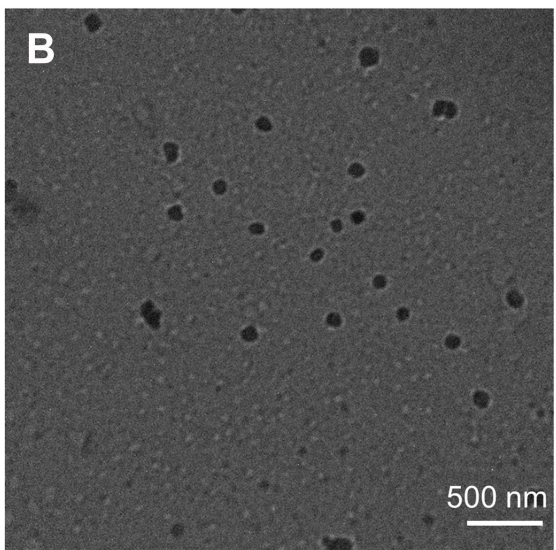

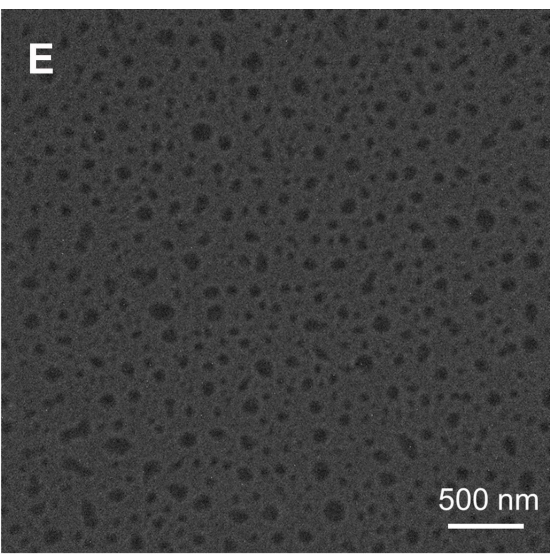

D

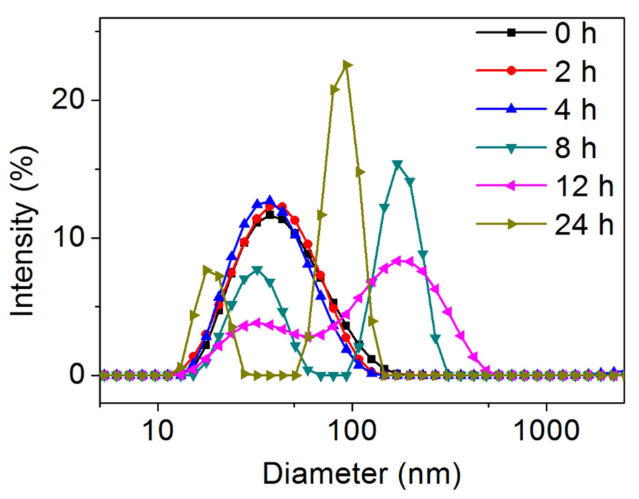

$\mathbf{F}$

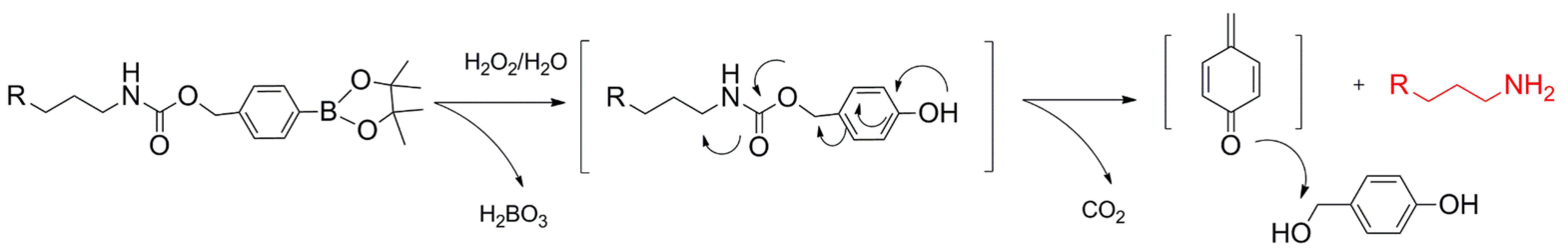

Figure I TEM images of (A) NPs and (B) NPs-CUR. (C) Hydrodynamic diameter distribution of NPs and NPs-CUR. Variation of the diameter distribution (D) and TEM image (E) of NPs treated with $100 \mathrm{mM} \mathrm{H}_{2} \mathrm{O}_{2}$ for $24 \mathrm{~h}$. (F) Proposed degradation mechanism of mPEG-b-P(PA-alt-GPBAe) by $\mathrm{H}_{2} \mathrm{O}_{2}$ and the oxidation products involved. 


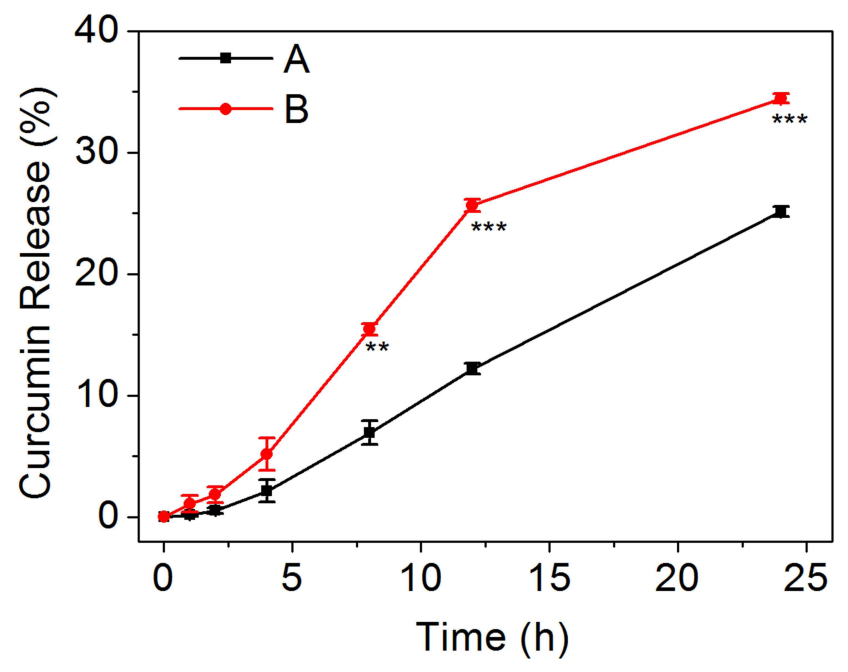

Figure 2 Cumulative drug release profiles of NPs-CUR in PBS at $\mathrm{pH} 7.4$ without $\mathrm{H}_{2} \mathrm{O}_{2}$ (A) or with $1.0 \mathrm{mM} \mathrm{H}_{2} \mathrm{O}_{2}$ (B). Data are presented as the average release percent \pm standard deviation $(n=3)$. $* * * P<0.001$ and $* * P<0.01$ were considered statistically significant.

mPEG- $b$-P(PA-alt-GPBAe) in NPs-CUR should be hydrophobic plus $\pi-\pi$ interaction. Both blank NPs and drugloaded NPs were solid, smooth, and spherical in shape, and the size was uniform (Figure 1A and B). DLS profiles demonstrated that the average diameter of the NPs was $40.2 \mathrm{~nm}$, and the polydispersity index (PDI) was 0.217 . In addition, NPs-CUR were slightly larger size $(49.4 \mathrm{~nm})$ and showed a broader distribution $(\mathrm{PDI}=0.336)$ compared with NPs (Figure 1C).

To verify the oxidation sensitivity of NPs, the size change of NPs in the presence of $100 \mathrm{mM} \mathrm{H}_{2} \mathrm{O}_{2}$ was monitored. This concentration is somewhat higher than the normal ROS level, and was used to prove the concept

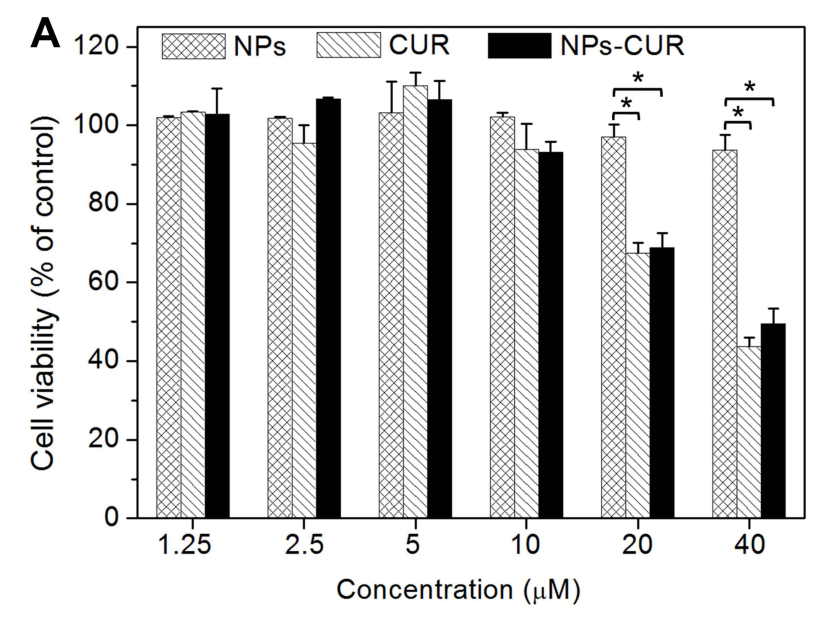

of ROS-degradability. As shown in Figure 1D, the size and distribution of the blank NPs did not change significantly after $4 \mathrm{~h}$, however, the DLS profile started to show two peaks after $8 \mathrm{~h}$. The extra peak indicated that some of the particles grew from $40 \mathrm{~nm}$ to $150-200 \mathrm{~nm}$, which was attributed to the swelling and dissociation of NPs upon treatment with $\mathrm{H}_{2} \mathrm{O}_{2}$. At $12 \mathrm{~h}$, the particle size distribution was the widest with a PDI value of 0.406 . Interestingly, after incubation in $\mathrm{H}_{2} \mathrm{O}_{2}$ solution for $24 \mathrm{~h}$, the diameter of the larger particles decreased, and the presence of peaks at $20 \mathrm{~nm}$ indicated that the original NPs were almost completely disrupted. The TEM image in Figure 1E demonstrates that after incubation in $\mathrm{H}_{2} \mathrm{O}_{2}$ solution for $24 \mathrm{~h}$, the sizes of the NPs varied, the structure became looser, and the edges were irregular. This could be explained by the proposed oxidation-degradation mechanism that is shown in Figure $1 F^{26,27}$ The hydrophobic phenylboronic pinacol ester groups reacted with $\mathrm{H}_{2} \mathrm{O}_{2}$ and underwent quinonemethide rearrangement, which resulted in hydrolysis of the carbamate linker, and finally the generation of hydrophilic $\mathrm{R}-\mathrm{NH}_{2}$ terminal groups. Thus, the NPs first swelled and disassociated $24 \mathrm{~h}$ later. This ROS-responsive behavior of NPs is useful for controlled drug release in a high oxidative environment, such as at sites of inflammation.

\section{In vitro Drug Release of NPs-CUR}

The in vitro drug release behavior of NPs-CUR is shown in Figure 2. In PBS without $\mathrm{H}_{2} \mathrm{O}_{2}$ (Group A), only $12.2 \%$ of CUR was gradually released in $12 \mathrm{~h}$, which was likely due to the physical diffusion of CUR from the hydrophobic core of NPs into PBS. In contrast, NPs exhibited

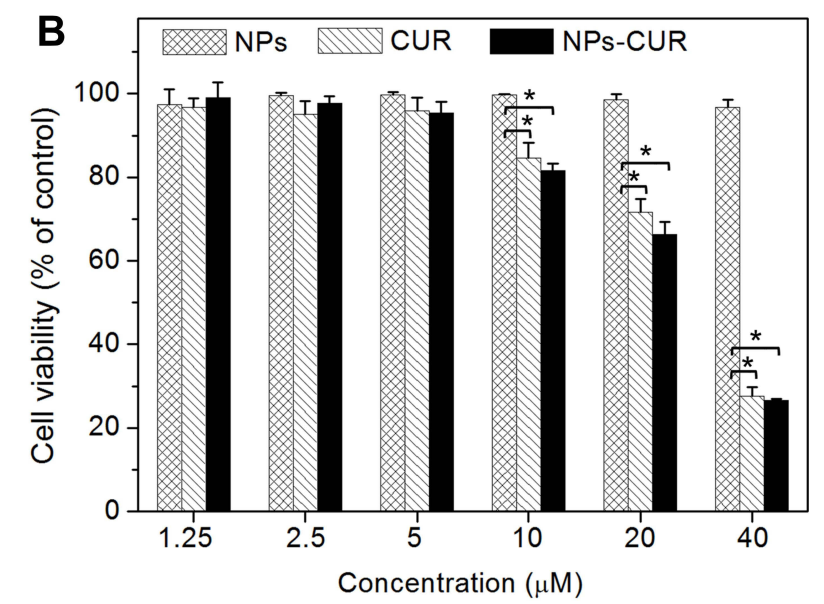

Figure 3 The in vitro cytotoxicity of NPs, CUR and NPs-CUR against (A) native and (B) LPS-stimulated RAW264.7 macrophages after incubation for 24 h. *P < 0.05 was considered statistically significant. 
a much faster drug release rate by trigger of $1.0 \mathrm{mM} \mathrm{H}_{2} \mathrm{O}_{2}$ (Group B), which resulted in $25.6 \%$ of CUR release in 12 $\mathrm{h}$, which was more than twice of that in Group A. The percentage of cumulative CUR release at $24 \mathrm{~h}$ was $25.1 \%$ and $34.4 \%$ for Group A and Group B, respectively. From the data presented in Figure 1D and E, we speculated that the oxidation-responsive drug release was attributed to a looser structure, a larger size distribution, and subsequently dissociation of the NPs after reaction with $\mathrm{H}_{2} \mathrm{O}_{2}$. Taken together, the results obtained in the in vitro drug release study confirmed the oxidation sensitivity of mPEG- $b$-P(PA-alt-GPBAe) NPs.

\section{Cytotoxicity and Cellular Uptake of NPs-CUR}

To examine the cytotoxicity towards native or LPS-activated RAW264.7 cells, free-form CUR, NPs, or NPs-CUR were incubated with RAW264.7 cells at a CUR dose of $1.25 \mu \mathrm{M}$ to $40 \mu \mathrm{M}$ for $24 \mathrm{~h}$; cells without any treatment were used as controls. The MTT assay results presented in Figure 3A and $B$ showed that the viability of cells in the NPs-treated group was above 90\%, indicating good biocompatibility of PEGylated alternating polyester copolymers at the test concentrations used. In contrast, dose-dependent cytotoxicity was observed for the groups treated with either free-form CUR or encapsulated CUR. The cell viability of the NPs-CUR group at a dose of $10 \mu \mathrm{M}$ was $\sim 85 \%$ and deceased to $\sim 68 \%$ when increasing the dose to $20 \mu \mathrm{M}$. In previous studies, it was demonstrated that low concentrations of CUR increased the level of ROS. ${ }^{28}$ Therefore, a moderate concentration of 10 $\mu \mathrm{M}$ CUR was chosen for the cell viability studies.

To monitor the ROS-insulting conditions of RAW264.7 macrophages, cells were incubated with various concentrations of $\mathrm{H}_{2} \mathrm{O}_{2}$ and incubated for $4 \mathrm{~h}$. Obvious cytotoxicity by $\mathrm{H}_{2} \mathrm{O}_{2}$ was observed in a dose-dependent manner (Figure $\underline{\mathrm{S} 5 \mathrm{~A}}$ ). However, treatment of $1 \mathrm{mM} \mathrm{H}_{2} \mathrm{O}_{2}$ (IC50 value), significantly increased the cell viability in the presence of NPs at concentrations from 0.063 to $0.50 \mathrm{mg} / \mathrm{mL}$ (Figure $\underline{\mathrm{S} 5 \mathrm{~B}}$ ), thereby indicating a protective effect of $\mathrm{mPEG}-b-\mathrm{P}$ (PA-alt-GPBAe) NPs to cells from oxidative damage.

The internalization of NPs was investigated by monitoring the green fluorescence of the loaded CUR using CLSM. A gradual increase of the fluorescence intensity from $2 \mathrm{~h}$ to $8 \mathrm{~h}$ revealed greater accumulation of CUR in RAW 264.7 cells and indicated a successful cellular uptake of NPs-CUR (Figure 4).

\section{In vitro Anti-Inflammatory Activity of NPs-CUR}

A well-defined model of macrophage activation at sites of inflammation involves treatment of RAW264.7 cells with LPS, which results in the overproduction of oxidants and irreversible oxidative cellular damage. We tested the antiinflammatory effects of NPs-CUR, free CUR, and NPs in this
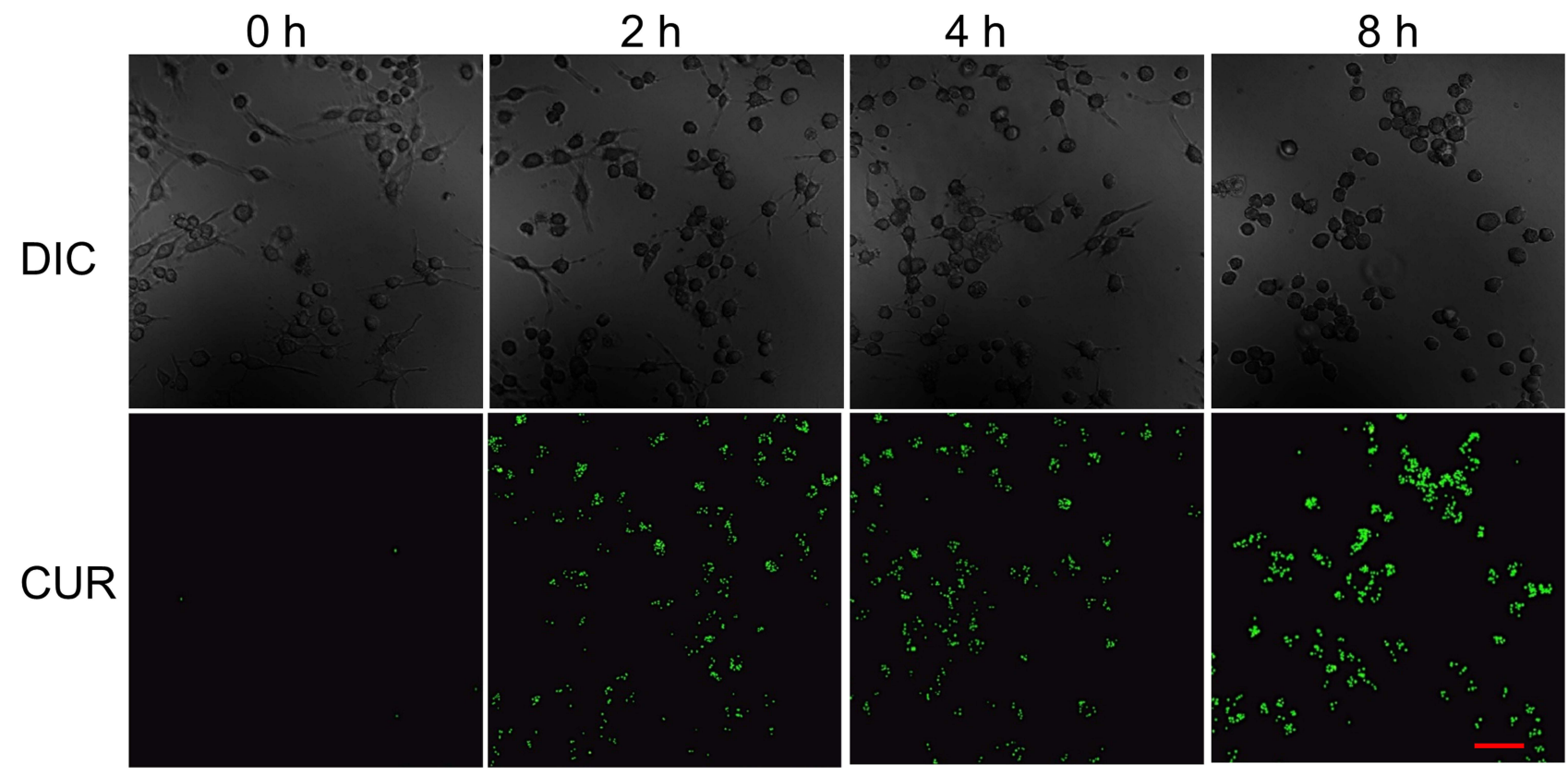

Figure 4 CLSM images of LPS-activated RAW 264.7 cells incubated with NPs-CUR for $2 \mathrm{~h}$ to $8 \mathrm{~h}$. Scale bars: $50 \mu \mathrm{m}$. 
LPS-stimulated macrophage model at a CUR dose of $10 \mu \mathrm{M}$. As shown in Figure 5A-C, the levels of pro-inflammatory cytokines TNF- $\alpha$, IL-1 $\beta$, and IL-6 were remarkably elevated in LPS-activated macrophages compared to non-activated controls $(\mathrm{P}<0.001)$, but were decreased to different degrees by all CUR-containing formulations. The average TNF- $\alpha$ concentration of NPs-CUR-treated cells was $284.5 \mathrm{ng} / \mathrm{L}$, which was far lower than that of untreated activated cells, ie, $426.7 \mathrm{ng} / \mathrm{L}(\mathrm{P}<0.001)$, and $18.6 \%$ lower than that of NPs groups ( $\mathrm{P}<0.05$ ). Unfortunately, no statistical differences of TNF- $\alpha$ levels were between free CUR and NPs-CUR-treated groups (Figure 5A). A similar inhibitory pattern was observed for IL-1 $\beta$ (Figure 5B). The average concentrations of IL-6 in NPs, free CUR, and NPs-CUR-treated groups were 88.2, 80.1, and $69.3 \mathrm{ng} / \mathrm{L}$, respectively (Figure 5C), which was significantly lower than that of control or LPS groups ( $p$ $<$ 0.05). Among pro-inflammatory cytokines, TNF- $\alpha$ secreted by activated macrophages plays a crucial role for the induction of other cytokines. The reduction of TNF- $\alpha$ secretion by our NPs-CUR formulation may promote the inhibition of IL-1 $\beta$ and IL-6 in LPS-stimulated RAW cells.

Apart from pro-inflammatory cytokines, the level of anti-inflammatory cytokine IL-10 was also evaluated. In our case, the increased levels of IL-10 were determined as follows: NPs-CUR $>$ NPs $>$ free CUR (Figure 5D). The best anti-inflammatory effect of NPs-CUR might be attributed to the protection of CUR during the blood circulation, and selective oxidation-triggered drug release at the inflammatory site.

\section{In vivo Anti-Inflammatory Activity of NPs-CUR}

To further evaluate the in vivo anti-inflammatory effect of NPs-CUR, a mouse model of acute ankle inflammation

C

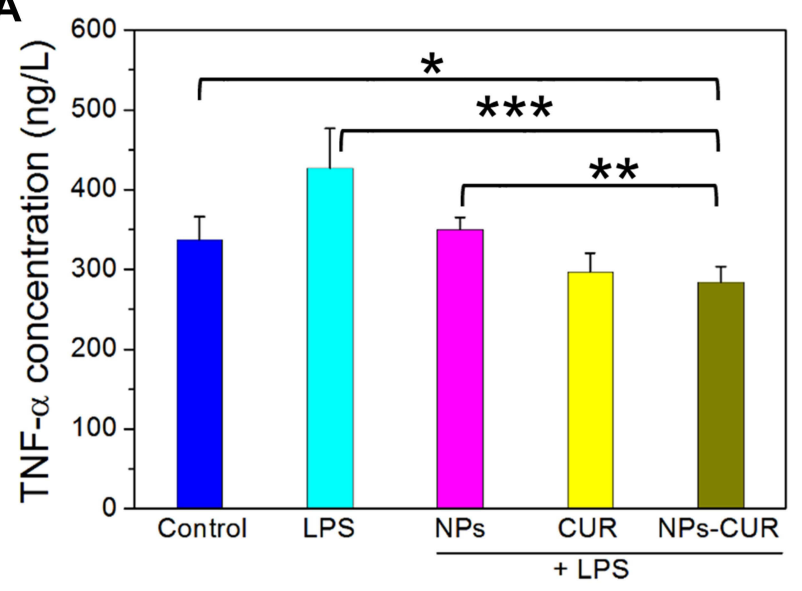

B

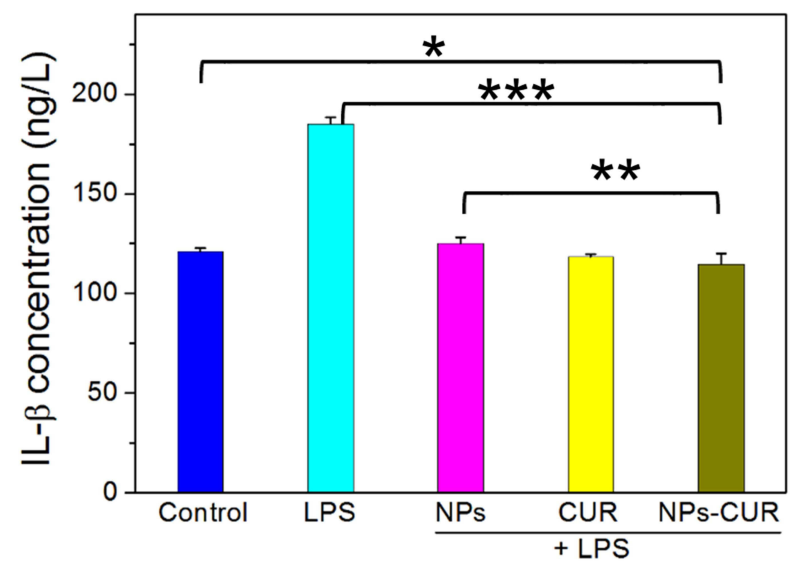

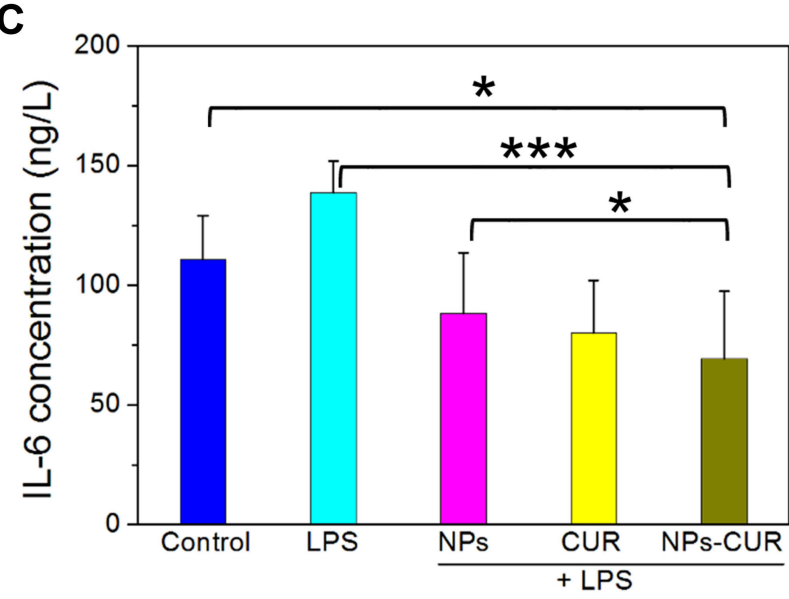

D

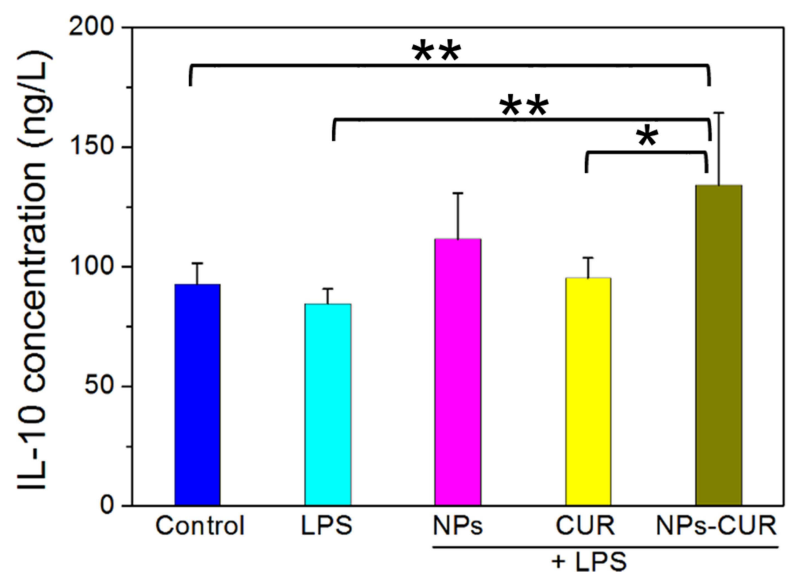

Figure 5 The concentrations of pro-inflammatory cytokines, including TNF- $\alpha$ (A), IL-I $\beta$ (B), IL-6 (C), and IL-I0 (D) in the supernatant of LPS) (I $\mu g / m L)$-stimulated RAW264.7 macrophages after treatment with NPs, CUR and NPs-CUR at a CUR dose of $10 \mu \mathrm{M}$. Cells treated with PBS without LPS stimulation were used as a control. Data are presented as the average \pm standard deviation $(n=5)$. $* P<0.05, * * P<0.01$ and $* * * P<0.001$ were considered statistically significant. 
was established by a local injection of LPS $(0.2 \mathrm{mg} / \mathrm{kg})$. Mice were treated with the same dose of PBS, NPs, free CUR, and NPs-CUR. Compared to the healthy ankle of the control group in Figure 6A, many inflammatory cells, which stained blue by hematoxylin, had infiltrated into the mouse ankle in the PBS-treated group, which was indicative of a success inflammation model induced by LPS. We also noticed that mice with an inflamed ankle mice moved more slowly than the healthy one.

Although the administration of free-form CUR could regulate inflammation by inhibiting pro-inflammatory cytokines in macrophages in vitro (Figure 5), a mild infiltration of inflammatory cells was observed in H\&E-stained sections of CUR-treated mice (Figure 6A). As expected, the most potent inflammatory inhibition was observed in mice treated with NPs-CUR compared with NPs and free drug. As shown in histopathological sections of the NPs-CUR group, very few inflammatory cells infiltrated in the affected ankle joint. Taken together, these results highlighted the importance of NPs carriers for in vivo delivery of CUR.

CD86 and CD 206 are typical surface markers for classically activated macrophages (M1) and alternatively activated macrophages (M2), respectively. ${ }^{29}$ As shown in Figure $6 \mathrm{~B}$ and $\mathrm{C}$, the lowest CD86 level and a maximum

A
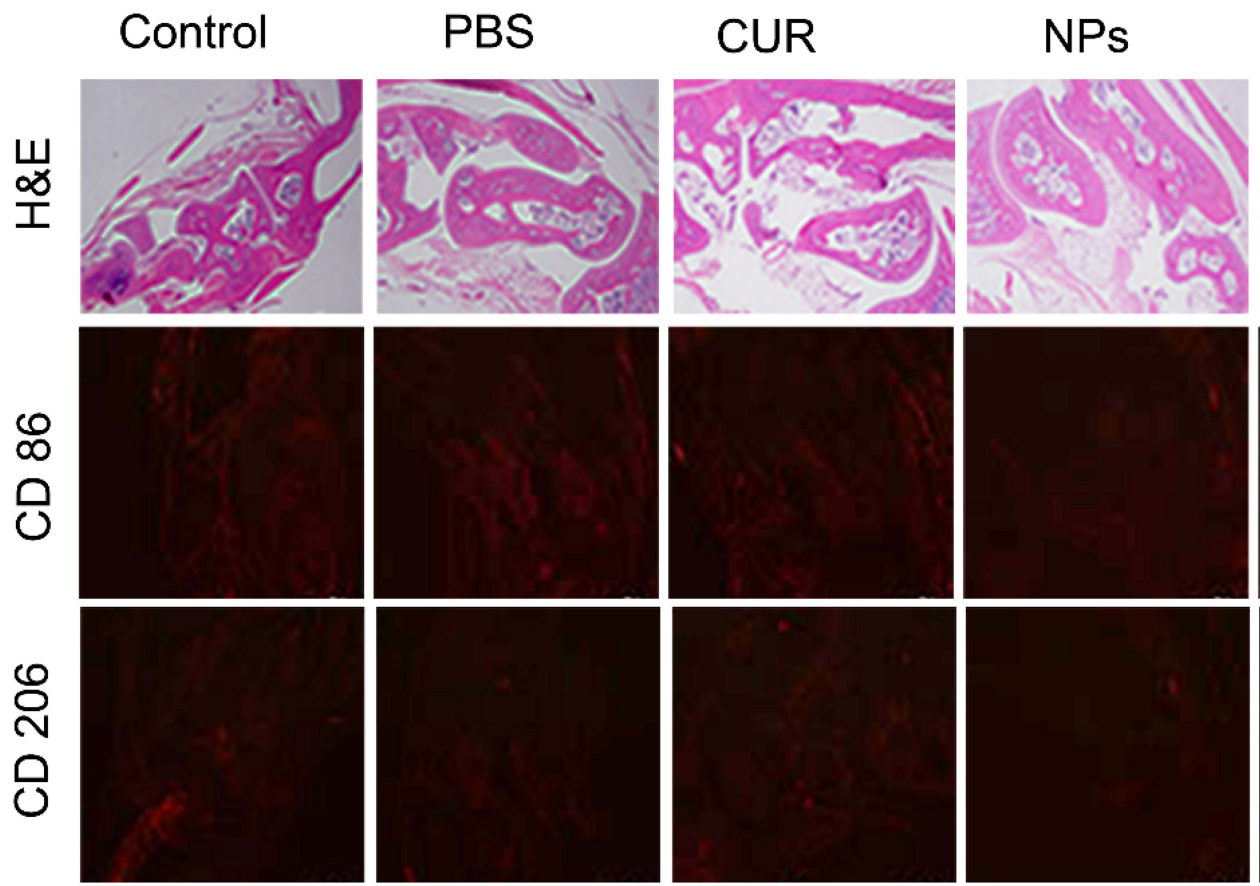

NPS-CUR

B
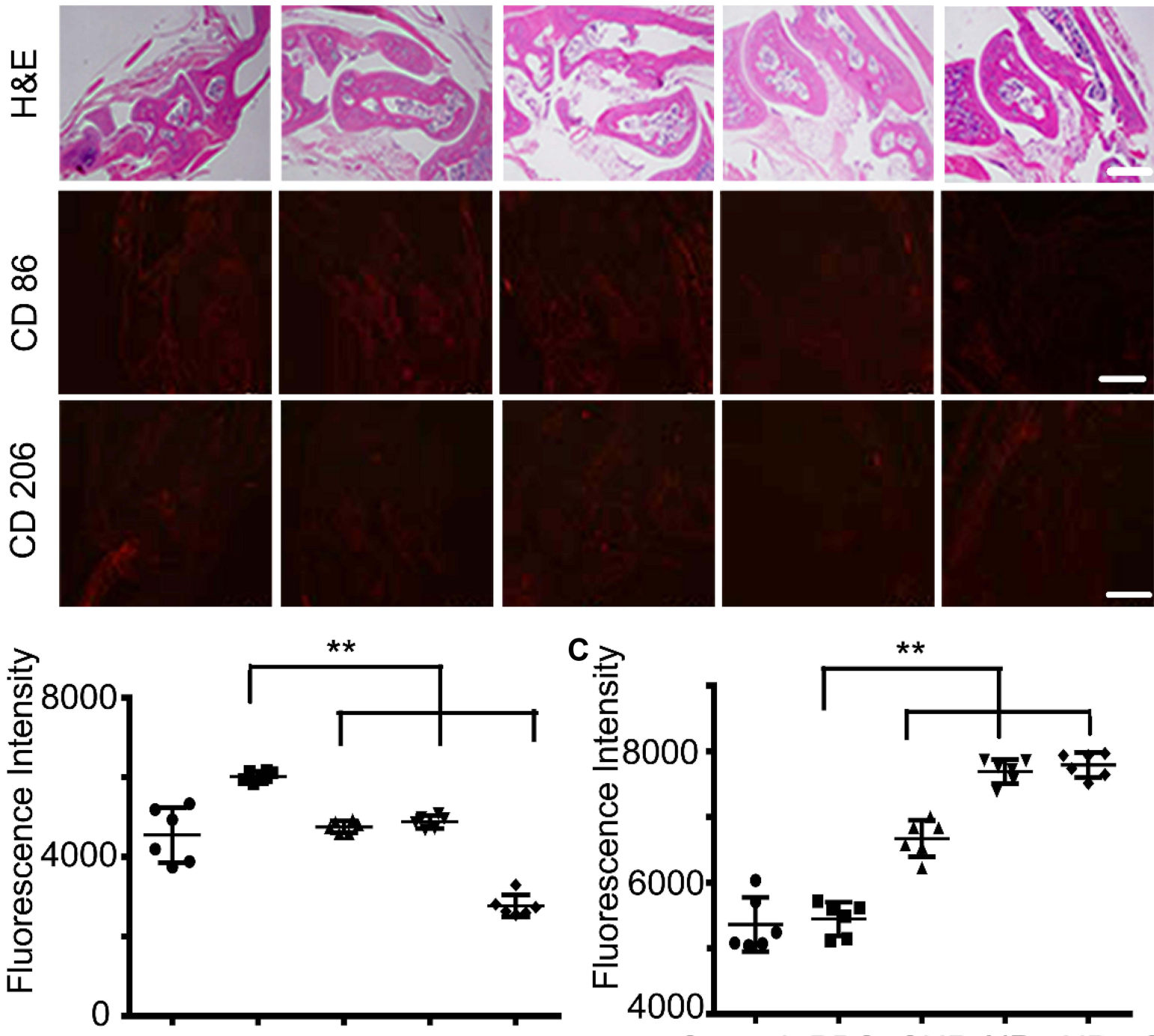

Control PBS CUR NPs NPs-CUR Control PBS CUR NPs NPs-CUR

Figure 6 (A) Microscopy images of H\&E and immunofluorescence analyses (CD 86 and CD 206) in a healthy mouse ankle (Control group), or LPS-infused ankles after treatment with PBS, NPs, free CUR or NPs-CUR at a CUR dose of $0.25 \mathrm{mg} / \mathrm{kg}$. Scale bar is $100.0 \mathrm{~mm}$. Statistical analysis by Image J for the fluorescence intensity of (B) CD 86 and $(C) C D$ 206, respectively. $* \mathrm{P}<0.05$ and $* * \mathrm{P}<0.01$ were considered statistically significant. 
A

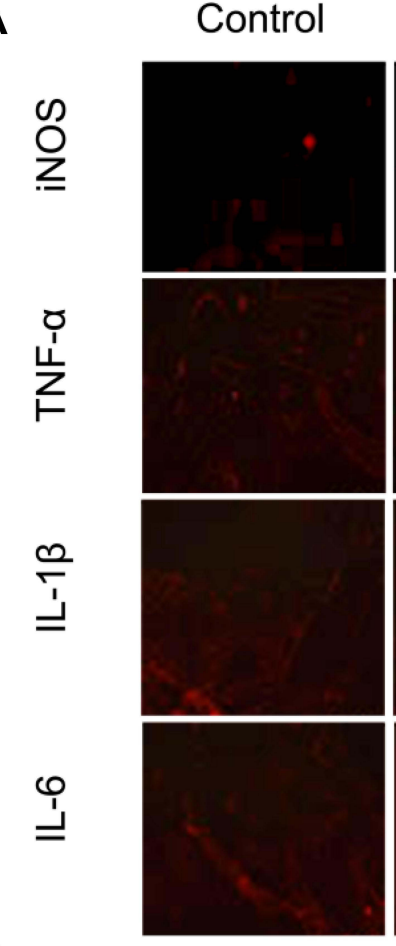

B

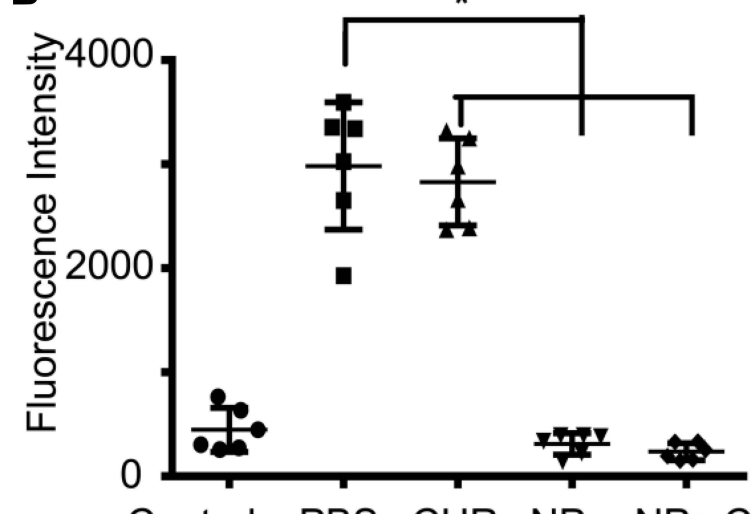

CUR
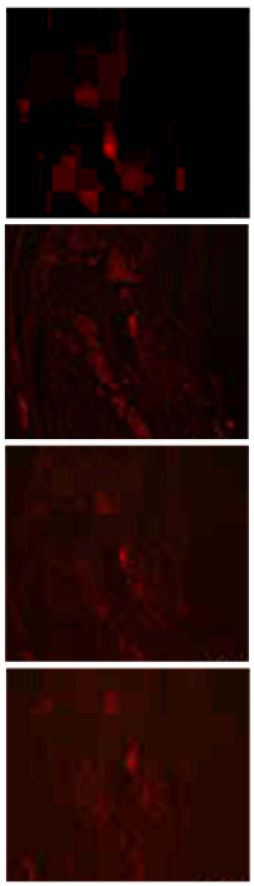

*

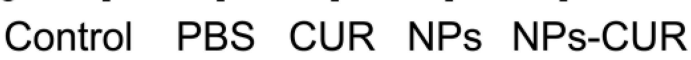

C

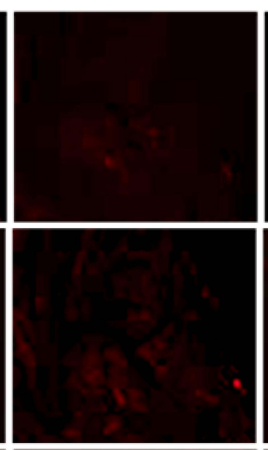

NPs

NPs-CUR
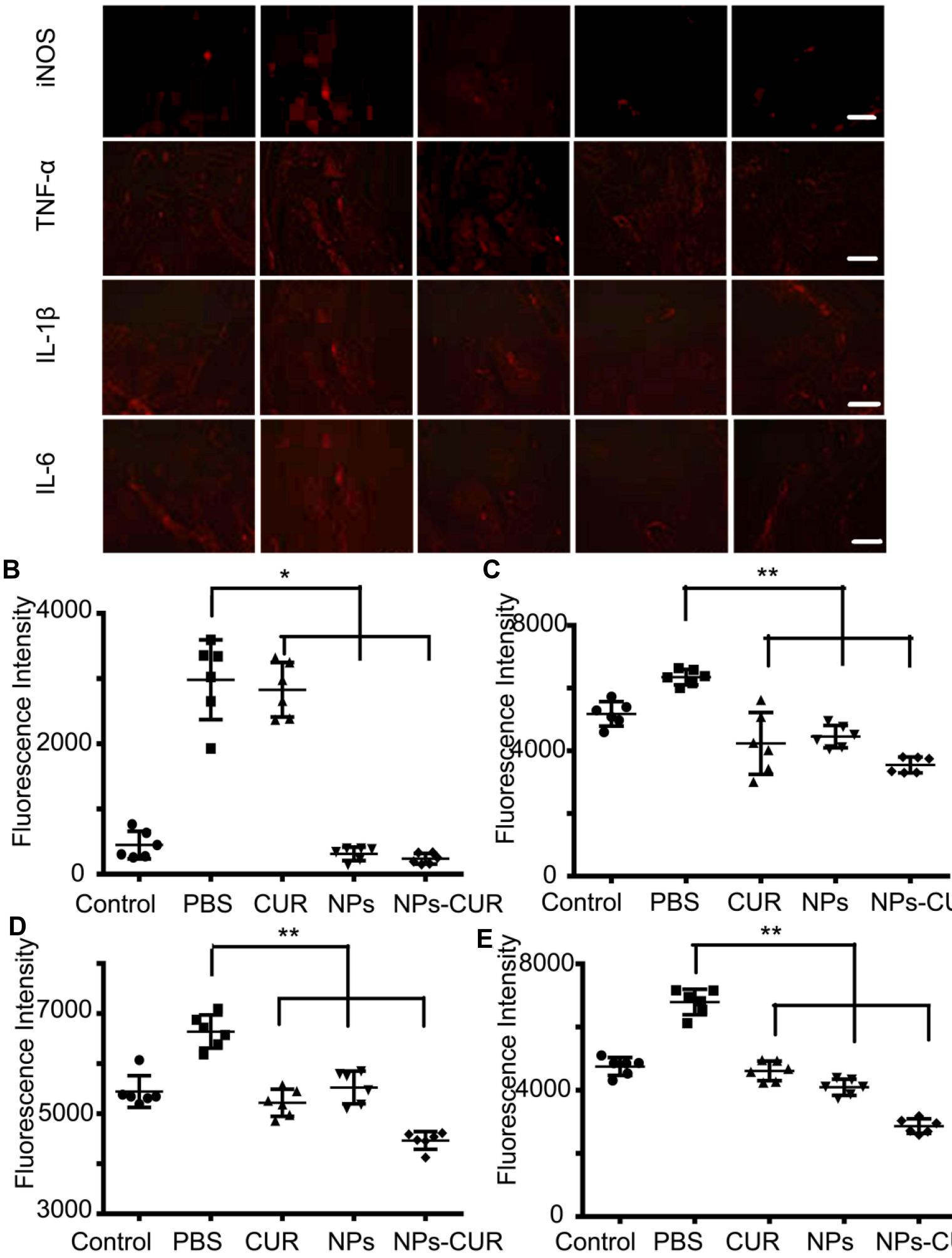

Control PBS CUR NPS NPS-CUR

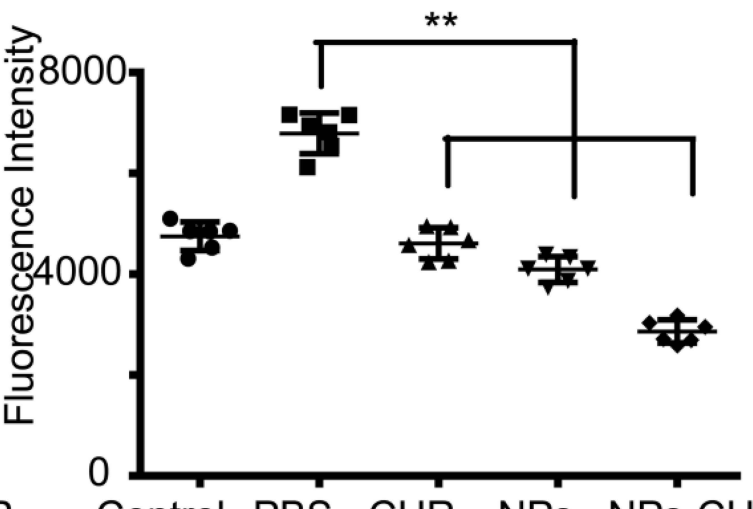

Figure 7 Immunofluorescence analyses of a healthy ankle (Control group) or LPS-affected ankles after treatment with PBS, NPs, free CUR or NPs-CUR. (A) Images with scale bar of $100.0 \mathrm{~mm}$. Statistical analysis by Image J for the fluorescence intensity of (B) iNOS, (C) TNF- $\alpha$, (D) IL-I $\beta$, and (E) IL-6). *P < 0.05 and $* * P<0.01$ were considered statistically significant. 
CD 206 intensity were observed in the ankle of the NPsCUR-treated group, which implied a potent ability of NPsCUR to modulate macrophage polarity from the proinflammatory M1 state to the anti-inflammatory M2 state. This was likely because the local ROS stress was reduced by NPs and the treatment by released CUR in the inflammatory site.

The levels of pro-inflammatory cytokines in the affected joints are key indicators of disease severity; consequently, immunofluorescence analysis of sections of inflamed ankle joints was performed to subsequently determine the differences in inflammatory conditions. As shown in Figure 7A, the expression of TNF- $\alpha$, IL- $1 \beta$, and IL-6 in the ankle sections was found to be increased in PBS-treated mice, but were reduced to different degrees after treatment with NPs, free CUR, or NPs-CUR compared with the healthy group. There were statistical differences of the cytokine levels between free-form CUR and NPs-CUR-treated groups $(\mathrm{P}<0.01$, Figure $7 \mathrm{C}-\mathrm{E})$. In addition, both pure NPs and NPs-CUR dramatically decreased the in vivo iNOS levels compared with PBS or CUR-treated groups (Figure 7B), which was indicative of the ROS-consumption ability and thus an inflammation inhibitory effect of polymeric NPs.

It is beyond doubt that the excessive generated ROS together with the pro-inflammatory cytokines, such as TNF- $\alpha$, IL-6, and IL-1 $\beta$ can cause significant damage to inflammatory cells. ${ }^{30}$ In this study, NPs composed with mPEG- $b$-P(PA-alt-GPBAe) reacted with $\mathrm{H}_{2} \mathrm{O}_{2}$ after cellular uptake, and reduced the oxidation stress of cells, and subsequently reduce the severity of inflammation. Subsequently, the NPs disassociated and the encapsulated CUR was selectively released at the inflammatory site (Scheme 1). It is well-established that CUR plays a critical role in controlling the signal transduction pathways involved in inflammatory responses, most likely through blocking of the nuclear transcription factor

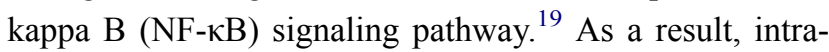
cellularly released CUR from NPs reduced the TNF- $\alpha$ production, and thus downregulated the expression of other cytokines, such as IL-1 $\beta$ and IL-6. Thus, both the in vitro and in vivo results indicated a significant inflammation regulatory effect of CUR treatment delivered by oxidation-sensitive polymeric NPs carriers.

\section{Conclusion}

In summary, ROS-sensitive NPs with an average diameter of $40.2 \mathrm{~nm}$ were obtained by self-assembly of a PEGylated polyester. The formulation, NPs-CUR, could effectively be triggered by $1 \mathrm{mM} \mathrm{H}_{2} \mathrm{O}_{2}$ to release the encapsulated CUR. Compared with NPs and free drug, NPs-CUR significantly inhibited the expression of pro-inflammatory cytokines, and markedly increased the production of anti-inflammatory cytokines both in vitro and in a murine model of LPS-induced ankle inflammation. Our results reveal that NPs carriers, which respond well to the low concentrations of $\mathrm{H}_{2} \mathrm{O}_{2}$ found in biological environments, exhibit enhanced anti-inflammatory activity of encapsulated drug. Future studies of our oxidation responsive NPs will involve the delivery of other clinical rapid-clearance anti-inflammatory agents to inflammatory sites, such as triamcinolone acetonide and dexamethasone, for the treatment of osteoarthritis.

\section{Acknowledgments}

We acknowledge the financial support from the National Natural Science Foundation of China (51803014, 52003268), and the Jilin Provincial Scientific and Technological Development Program (20200801006GH, 20200201254JC). The author would like to thank the support from the Education Department of Jilin Province (Grant number JJKH20200744KJ).

\section{Disclosure}

The authors report no conflicts of interest in this work.

\section{References}

1. Khandelwal PJ, Herman AM, Moussa CEH. Inflammation in the early stages of neurodegenerative pathology. J Neuroimmunol. 2011;238 (1):1-11. doi:10.1016/j.jneuroim.2011.07.002

2. Basu S. Bioactive eicosanoids: role of prostaglandin F2 $\alpha$ and F2isoprostanes in inflammation and oxidative stress related pathology. Mol Cells. 2010;30(5):383-391. doi:10.1007/s10059-010-0157-1

3. Mehta P, McAuley DF, Brown M, Sanchez E, Tattersall RS, Manson JJ. COVID-19: consider cytokine storm syndromes and immunosuppression. Lancet. 2020;395(10229):1033-1034. doi:10.1016/S0140-6736(20)30628-0

4. Merad M, Martin JC. Pathological inflammation in patients with COVID-19: a key role for monocytes and macrophages. Nat Rev Immunol. 2020;20(6):355-362. doi:10.1038/s41577-020-0331-4

5. Tian H, Zhang M, Jin J, Jiang Y, Luan Y. Cu-MOF chemodynamic nanoplatform via modulating glutathione and $\mathrm{H} 2 \mathrm{O} 2$ in tumor microenvironment for amplified cancer therapy. $J$ Colloid Interface Sci. 2020;587:358-366. doi:10.1016/j.jcis.2020.12.028

6. Pu HL, Chiang WL, Maiti B, et al. Nanoparticles with dual responses to oxidative stress and reduced $\mathrm{ph}$ for drug release and anti-inflammatory applications. ACS Nano. 2014;8(2):1213-1221. doi: $10.1021 / \mathrm{nn} 4058787$

7. Lallana E, Tirelli N. Oxidation-responsive polymers: which groups to use, how to make them, what to expect from them (Biomedical Applications). Macromol Chem Phys. 2013;214(2):143-158. doi:10.1002/macp.201200502 
8. Xiaoya DYW, Gaoli L, Xiao C, Chen X. Iminoboronate ester cross-linked hydrogels with injectable, self-healing and multi-responsive properties. Acta Polym Sin. 2019;50(5):505-515.

9. Kwon J, Kim J, Park S, Khang G, Kang PM, Lee D. Inflammationresponsive antioxidant nanoparticles based on a polymeric prodrug of vanillin. Biomacromolecules. 2013;14(5):1618-1626. doi:10.1021/ bm400256h

10. Park S, Yoon J, Bae S, et al. Therapeutic use of H2O2-responsive anti-oxidant polymer nanoparticles for doxorubicin-induced cardiomyopathy. Biomaterials. 2014;35(22):5944-5953. doi:10.1016/j.biomaterials.2014.03.084

11. Ko E, Jeong D, Kim J, Park S, Khang G, Lee D. Antioxidant polymeric prodrug microparticles as a therapeutic system for acute liver failure. Biomaterials. 2014;35(12):3895-3902. doi:10.1016/j. biomaterials.2014.01.048

12. Napoli A, Valentini M, Tirelli N, Müller M, Hubbell JA. Oxidationresponsive polymeric vesicles. Nat Mater. 2004;3(3):183-189. doi: $10.1038 /$ nmat 1081

13. El Mohtadi F, d'Arcy R, Yang X, Turhan ZY, Alshamsan A, Tirelli N. Main chain polysulfoxides as active 'Stealth' polymers with additional antioxidant and anti-inflammatory behaviour. Int $\mathrm{J} \mathrm{Mol} \mathrm{Sci.}$ 2019;20(18):18. doi:10.3390/ijms20184583

14. Rajkovic O, Gourmel C, d'Arcy R, et al. Reactive oxygen species-responsive nanoparticles for the treatment of ischemic stroke. Adv Ther. 2019;2(7):1900038. doi:10.1002/adtp.201900038

15. Tapeinos C, Pandit A. Physical, chemical, and biological structures based on ROS-sensitive moieties that are able to respond to oxidative microenvironments. Adv Mater. 2016;28(27):5553-5585. doi:10.1002/adma.201505376

16. Trummer M, Choffat F, Smith P, Caseri W. Polystannanes: synthesis, properties, and outlook. Macromol Rapid Commun. 2012;33(67):448-460. doi:10.1002/marc.201100794

17. Herzberger J, Fischer K, Leibig D, Bros M, Thiermann R, Frey H. Oxidation-responsive and "Clickable" poly(ethylene glycol) via copolymerization of 2-(methylthio)ethyl glycidyl ether. $J$ Am Chem Soc. 2016;138(29):9212-9223. doi:10.1021/jacs.6b04548

18. Esatbeyoglu T, Huebbe P, Ernst IMA, Chin D, Wagner AE, Rimbach G. Curcumin-From molecule to biological function. Angew Chem Int Ed. 2012;51(22):5308-5332. doi:10.1002/ anie. 201107724

19. Banerjee S, Chakravarty AR. Metal complexes of curcumin for cellular imaging, targeting, and photoinduced anticancer activity. Acc Chem Res. 2015;48(7):2075-2083. doi:10.1021/acs. accounts. 5 b00127
20. Chowdhury MR, Moshikur RM, Wakabayashi R, et al. Development of a novel ionic liquid-curcumin complex to enhance its solubility, stability, and activity. Chem Commun. 2019;55(54):7737-7740. doi:10.1039/C9CC02812A

21. Plavcová Z, Šalamúnová P, Saloň I, Štěpánek F, Hanuš J, Hošek J. Curcumin encapsulation in yeast glucan particles promotes its anti-inflammatory potential in vitro. Int J Pharm. 2019;568:118532. doi:10.1016/j.ijpharm.2019.118532

22. Tomren MA, Másson M, Loftsson T, Tønnesen HH. Studies on curcumin and curcuminoids: XXXI. Symmetric and asymmetric curcuminoids: stability, activity and complexation with cyclodextrin. Int J Pharm. 2007;338(1):27-34. doi:10.1016/j.ijpharm.2007.01.013

23. Zhang Y, Zhang H, He P, Yi X, Liu X, Xiao C. A PEGylated alternating copolymeric prodrug of sulfur dioxide with glutathione responsiveness for Irinotecan delivery. $J$ Mat Chem B. 2021;9 (1):187-194. doi:10.1039/D0TB02097D

24. Zhang Y, He P, Liu X, et al. Synthesis of PEGylated alternating copolymer bearing thioether pendants for oxidation responsive drug delivery. Eur Polym J. 2018;107:308-314. doi:10.1016/j. eurpolymj.2018.09.004

25. Zhang Y, He P, Liu X, et al. A PEGylated alternating copolymer with oxidation-sensitive phenylboronic ester pendants for anticancer drug delivery. Biomat Sci. 2019;7(9):3898-3905. doi:10.1039/ C9BM00884E

26. Broaders KE, Grandhe S, Fréchet JMJ. A biocompatible oxidation-triggered carrier polymer with potential in therapeutics. $J$ Am Chem Soc. 2011;133(4):756-758. doi:10.1021/ja110468v

27. Song -C-C, Du F-S, Li Z-C. Oxidation-responsive polymers for biomedical applications. J. Mater. Chem. B. 2014;2(22):3413-3426. doi:10.1039/C3TB21725F

28. Strasser E-M, Wessner B, Manhart N, Roth E. The relationship between the anti-inflammatory effects of curcumin and cellular glutathione content in myelomonocytic cells. Biochem Pharmacol. 2005;70(4):552-559. doi:10.1016/j.bcp.2005.05.030

29. Farajzadeh R, Zarghami N, Serati-Nouri H, et al. Macrophage repolarization using CD44-targeting hyaluronic acid-polylactide nanoparticles containing curcumin. Artif Cells, Nanomed Biotechnol. 2018;46 (8):2013-2021.

30. Luo W, Wang Y, Lin F, et al. Selenium-doped carbon quantum dots efficiently ameliorate secondary spinal cord injury via scavenging reactive oxygen species. Int $J$ Nanomedicine. 2020;15:10113-10125. doi:10.2147/IJN.S282985
International Journal of Nanomedicine

\section{Publish your work in this journal}

The International Journal of Nanomedicine is an international, peerreviewed journal focusing on the application of nanotechnology in diagnostics, therapeutics, and drug delivery systems throughout the biomedical field. This journal is indexed on PubMed Central, MedLine, CAS, SciSearch ${ }^{\mathbb{R}}$, Current Contents ${ }^{\mathbb{R}} /$ Clinical Medicine, $^{2}$
Journal Citation Reports/Science Edition, EMBase, Scopus and the Elsevier Bibliographic databases. The manuscript management system is completely online and includes a very quick and fair peer-review system, which is all easy to use. Visit http://www.dovepress.com/ testimonials.php to read real quotes from published authors. 